\title{
Fault-Tolerant Multiagent Robotic Formation Control Exploiting System Symmetries
}

\author{
Bill Goodwine and Panos Antsaklis
}

\begin{abstract}
This paper extends some prior work by the authors to address general robustness of solutions in multiagent coordination control problems. In particular, it focuses on fault-tolerant formation control. In our prior work, symmetries in the system were exploited to simplify the nonlinear Lyapunov stability analysis for symmetric systems. The type of symmetry considered is a discrete symmetry where the system is composed of many repeated instances of interacting identical agents. The results are based on Lyapunov methods, and hence are of general applicability and specifically these results are applicable to both distributed as well as non-distributed coordination methods. This paper considers the same types of systems and extends the stability results to the cases of robustness of formation stability under failure of individual agents.
\end{abstract}

\section{INTRODUCTION}

This paper considers fault-tolerant formation control for multi-agent systems. Formation control for multiple mobile robotic systems has a long history, with the main focus being on the use of potential functions for coordination (see for example [1], [2], [3], [4], [5], [6], [7] and the citations therein). The use of potential functions has an obvious appeal in that they facilitate stability analyses using Lyapunov functions. The drawbacks are well-known also, which include the existence of multiple local minima in complex environments, the fact that realistic potential functions representing the realities of sensor ranges introduce mathematical limitations on the potential functions which complicate and limit the stability analysis, etc. As observed in [8], many of the prior efforts have assumed specific dynamics with the correct observation that they probably generalize. Motivated by this, our approach has been to develop a general formulation which is the foundational framework underlying many of the results in the literature that focus on specific system dynamics.

The contributions of our prior work in [9] included 1) a nonlinear extension of the results in [10], [11] which is a more straight-forward representation of system symmetries than our previous work; 2) the presentation of a unified theoretical framework that is underlying many of the formation control algorithms in the literature; and, 3) a general stability theorem that is applicable to such systems regardless of the number of components. These results allow a control design

The support of the National Science Foundation under Grant No. CNS1035655 is gratefully acknowledged.

Bill Goodwine is with the Department of Aerospace \& Mechanical Engineering, University of Notre Dame, Notre Dame, Indiana 46556 USA goodwine@controls. ame.nd.edu

Panos Antsaklis is with the Department of Electrical Engineering at the University of Notre Dame, Notre Dame, Indiana 46556 USA pantsaklend.edu

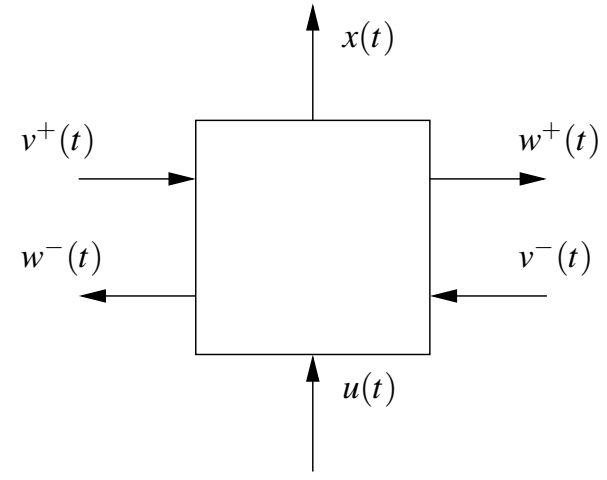

Fig. 1. System building block in one spatial dimension.

engineer to focus the analysis on a smaller, more tractable system with a guarantee that stability conclusions will hold for a much larger system. This paper extends those results to determine conditions under which a formation is still stable (and in what sense it is stable) when individual agents fail.

Some of our prior work [12], [13], [14], [15] considers system symmetries that are defined by a group action on the configuration manifold for a distributed system that was induced by the action of a permutation group. The main drawback of such an approach is that, in the general case, identifying such symmetries can be problematic. However, in the case of most engineering and robotics systems, where the individual robots are the components that are symmetric, symmetry identification is much less of a problem. Rather than using this prior approach, this paper is based on our more straight-forward approach from [9]. However, it is emphasized that the prior approaches [12], [13], [14], [15], [16] offer a general approach to the problem that can be used in cases more general than the ones addressed here.

This paper is organized as follows. Section II defines the types of systems we consider, specifically symmetric systems and symmetric systems with periodic interconnections. The main result appears in Section III, which is a proposition that provides conditions for the (asymptotic) stability of a formation when one or more of the robots in the formation fail. An example from the literature with second order dynamics is developed throughout the paper to illustrate the application of the definition and result. Simulations are also included to verify the theoretical results. Section IV presents the conclusions and avenues of future work.

\section{SyMmetriC SyStemS}

The "basic building block" in one spatial dimension is illustrated in Figure 1. The outputs from the component are 
$w^{-}(t)$ and $w^{+}(t)$, and the inputs are $u, v^{-}(t)$ and $v^{+}(t)$. The signals $v^{ \pm}$represent the effects of the coupling with the other components and $u$ are the usual control inputs which need to be designed for stability, performance, robustness, etc.

We wish to express component-by-component, the usual dynamics of a nonlinear control system expressed by

$$
\dot{x}=f(x)+\sum_{j=1}^{m} g_{j}(x) u_{j},
$$

where $x \in \mathbb{R}^{n}$ and the vector fields $f(x), g_{j}(x) \in T \mathbb{R}^{n}$. In the general case, the vector fields $f$ and $g_{j}$ depend on the coupling with the other components, so the equations governing the dynamics of this component are given by

$$
\begin{aligned}
\dot{x}_{i}(t)= & f_{i}\left(x_{i}(t), v_{i}^{+}(t), v_{i}^{-}(t)\right) \\
& +\sum_{j=1}^{m} g_{j, i}\left(x_{i}(t), v_{i}^{+}(t), v_{i}^{-}(t)\right) u_{j}(t) \\
w_{i}^{-}(t)= & \hat{f}_{i}^{-}\left(x_{i}(t), v_{i}^{+}(t), v_{i}^{-}(t)\right), \\
w_{i}^{+}(t)= & \hat{f}_{i}^{+}\left(x_{i}(t), v_{i}^{+}(t), v_{i}^{-}(t)\right) .
\end{aligned}
$$

For a system of interconnected components where the incoming signals, $v^{ \pm}(t)$ are from the outgoing signals from the component's neighbors, the entire system is coupled because the $w^{ \pm}(t)$ signals depend on the component's input signals, $v^{ \pm}(t)$. The class of the types of coupling that could be represented by this formulation is very broad and could include, for example, when there is a physical joining of agents, as with reconfigurable, modular robots.

For mobile robots where there is no physical contact between the robots and the topology of the structure of the system is such that a component only receives a limited number of inputs (from its nearest neighbors, for example), the nature of the coupling between the robots can be expressed in a simpler manner. In particular, it is only through the control inputs that the output from the other components affects the dynamics of an agent, which is expressed by

$$
\begin{aligned}
\dot{x}_{i}(t) & =f_{i}\left(x_{i}(t)\right)+\sum_{j=1}^{m} g_{j, i}\left(x_{i}(t)\right) u_{j, i}(t) \\
w_{i}^{-}(t) & =\hat{f}_{i}^{-}\left(x_{i}(t)\right), \quad w_{i}^{+}(t)=\hat{f}_{i}^{+}\left(x_{i}(t)\right),
\end{aligned}
$$

where writing

$$
u_{j, i}(t)=u_{j, i}\left(x_{i}(t), v_{i}^{+}(t), v_{i}^{-}(t)\right)
$$

makes the coupling of the agent dynamics through feedback in the input explicit.

We can build up a system with $N$ components by requiring periodic interconnections in one dimension, i.e.,

$$
v_{i+1}^{+}(t)=w_{i}^{+}(t), \quad v_{i-1}^{-}(t)=w_{i}^{-}(t),
$$

where all mathematical operations on indices are $\bmod (N)$. A segment from such a periodically interconnected system is illustrated in Figure 2. In this case, feedback can be expressed in terms of the outputs from the neighbors and Equation 2 can be written as

$$
u_{j, i}=u_{j, i}\left(x_{i}(t), w_{i-1}^{+}\left(x_{i-1}(t)\right), w_{i+1}^{-}\left(x_{i+1}(t)\right) .\right.
$$

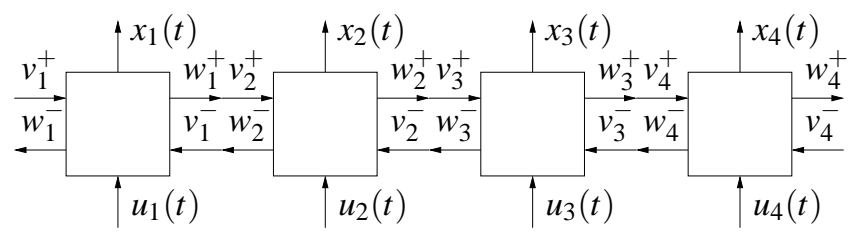

Fig. 2. System interconnected in one spatial dimension.

The simplest type of symmetric system would be when all the components are identical with periodic interconnections, and example of which would be a fleet of autonomous robots that are in a formation where each robot communicates in an identical manner to its two nearest neighbors.

Definition 1 Consider a system of the form of Equation 1. This system is a symmetric system spatially connected in one dimension if it has periodic interconnections and

$$
\begin{aligned}
f_{i}(x) & =f_{k}(x), & g_{j, i}(x) & =g_{j, k}(x), \\
\hat{f}_{i}^{-}(x) & =\hat{f}_{k}^{-}(x), & \hat{f}_{i}^{+}(x) & =\hat{f}_{k}^{+}(x)
\end{aligned}
$$

for $x \in \mathbb{R}^{n}$, for all $i, k=1, \ldots, N$ and for each $j=1, \ldots, m$. A system under state feedback is a feedback symmetric system if it is a symmetric system and

$$
u_{j, i}\left(x_{1}, w_{i-1}^{+}\left(x_{2}\right), w_{i+1}^{-}\left(x_{3}\right)\right)=u_{j, k}\left(x_{1}, w_{k-1}^{+}\left(x_{2}\right), w_{k+1}^{-}\left(x_{3}\right)\right)
$$

for $\left(x_{1}, x_{2}, x_{3}\right) \in \mathbb{R}^{n} \times \mathbb{R}^{n} \times \mathbb{R}^{n}$, for all $i, k=1, \ldots, N$ and for each $j=1, \ldots, m$.

To allow more general topologies than connections in one spatial dimension, define the index sets $\mathcal{V}_{i}$ and $\mathcal{W}_{i}$ which index multiple inputs and outputs respectively for component $i$. The outputs from component $i$ are denoted by $w_{i}^{l}(t), l \in \mathcal{W}_{i}$ and the inputs are denoted by $v_{i}^{l}(t), l \in \mathcal{V}_{i}$.

The dynamics of a component are given by

$$
\begin{aligned}
\dot{x}_{i}(t) & =f_{i}\left(x_{i}(t)\right)+\sum_{j=1}^{m} g_{j, i}\left(x_{i}(t)\right) u_{j}(t) \\
w_{i}^{l}(t) & =\hat{f}_{i}^{l}\left(x_{i}(t)\right), \quad \forall l \in \mathcal{W}_{i}
\end{aligned}
$$

with

$$
u_{j, i}(t)=u_{j, i}\left(x_{i}(t), v_{i}^{l_{1}}(t), v_{i}^{l_{2}}(t), v_{i}^{l_{3}}(t), \ldots\right)
$$

where $l_{1}, l_{2}, \ldots \in \mathcal{V}_{i}$.

Periodic interconnections are defined in a manner similar to the case of one spatial dimension. In particular, if there exist orderings of each of the sets $\mathcal{V}_{i}$ and $\mathcal{W}_{i}$ and

$$
v_{i}^{j}(t)=w_{j}^{i}(t) \quad \forall j \in \mathcal{V}_{i}, \forall i \in \mathcal{W}_{j}
$$

then the system has periodic interconnections, which requires that $\mathcal{V}_{i}$ and $\mathcal{W}_{i}$ have the same size. For periodic interconnections, the input under feedback can be written as

$$
u_{j, i}(t)=u_{j, i}\left(x_{i}(t), w_{i}^{l_{1}}\left(x_{l_{1}}(t)\right), w_{i}^{l_{2}}\left(x_{l_{2}}(t)\right), w_{i}^{l_{3}}\left(x_{l_{3}}(t)\right), \ldots\right) .
$$

for $l_{1}, l_{2}, \ldots, \in \mathcal{W}_{i}$. 


\section{A. Equivalent Symmetric Systems}

Finally, we want to "build up" a system. An $N+1$ component system will be equivalent to the $N$ component system if

$$
f_{i}(x)=f_{k}(x), \quad g_{j, i}(x)=g_{j, k}(x)
$$

for $x \in \mathbb{R}^{n}$, for all $k=1, \ldots, N$ and for all $i=1, \ldots, N+1$ and for each $j=1, \ldots, m$ and there exists an ordering for each $\mathcal{W}_{i}$ such that $\hat{f}_{i}^{j}(x)=\hat{f}_{k}^{l}(x)$ for $j \in \mathcal{W}_{i}$ and $l \in \mathcal{W}_{k}$. The $N+1$ system is feedback equivalent to the $N$ system if

$$
\begin{gathered}
u_{j, i}\left(x_{i}(t), w_{l_{1}}^{i}\left(x_{l_{1}}(t)\right), w_{l_{2}}^{i}\left(x_{l_{2}}(t)\right), w_{l_{3}}^{i}\left(x_{l_{3}}(t)\right), \ldots\right)= \\
u_{j, k}\left(x_{k}(t), w_{m_{1}}^{k}\left(x_{m_{1}}(t)\right), w_{m_{2}}^{k}\left(x_{m_{2}}(t)\right), w_{m_{3}}^{k}\left(x_{m_{3}}(t)\right), \ldots\right)
\end{gathered}
$$

for $\left(x_{1}, x_{2}, x_{3}\right) \in \mathbb{R}^{n} \times \mathbb{R}^{n} \times \mathbb{R}^{n}$, for all $k=1, \ldots, N, i=$ $1, \ldots, N+1$, for each $j=1, \ldots, m$, for $l_{1}, l_{2}, \ldots, \in \mathcal{W}_{i}$ and for $m_{1}, m_{2}, \ldots, \in \mathcal{W}_{k}$.

For notational convenience, we will stack all the states and vector fields from each component into one system description, $\dot{x}=f(x)+g(x) u(t)$ where

$x=\left[\begin{array}{c}x_{1} \\ x_{2} \\ \vdots \\ x_{N}\end{array}\right], u=\left[\begin{array}{c}u_{1} \\ u_{2} \\ \vdots \\ u_{N}\end{array}\right], f(x)=\left[\begin{array}{c}f_{1}\left(x_{1}\right) \\ f_{2}\left(x_{2}\right) \\ \vdots \\ f_{N}\left(x_{N}\right)\end{array}\right], g(x)=\left[\begin{array}{c}g_{1}\left(x_{1}\right) \\ g_{2}\left(x_{2}\right) \\ \vdots \\ g_{N}\left(x_{N}\right)\end{array}\right]$.

Example 1 A recurring example in this paper will be planar agents with second order dynamics used in [3]. Each robot has a location and velocity in $\mathbb{R}^{2}$, with equations of motion for the $i$ th robot given by

$$
\frac{d}{d t}\left[\begin{array}{c}
x_{i} \\
\dot{x}_{i} \\
y_{i} \\
\dot{y}_{i}
\end{array}\right]=\left[\begin{array}{c}
\dot{x}_{i} \\
0 \\
\dot{y}_{i} \\
0
\end{array}\right]+\left[\begin{array}{l}
0 \\
1 \\
0 \\
0
\end{array}\right] u_{1, i}+\left[\begin{array}{l}
0 \\
0 \\
0 \\
1
\end{array}\right] u_{2, i} .
$$

The goal formation is a regular $\mathrm{N}$-polygon and each robot communicates with its two neighbors and the next two agents, as is illustrated in Figure 3. In Figure 3, each node in the graph is a component similar to that illustrated in Figure 1; however, in this case each component has four outputs, each of which is simply its position and similarly each node has four inputs which are the outputs of the connected components, i.e.,

$$
w_{i}^{j}=\left[\begin{array}{l}
x_{i} \\
y_{i}
\end{array}\right], \quad v_{i}^{j}=\left[\begin{array}{l}
x_{j} \\
y_{j}
\end{array}\right]
$$

where $j \in\{i-2, i-i, i+1, i+2\}$. In the figure, each arrow represents both an input and output.

Let the desired distance between components $i$ and $j$ be

$$
d_{i j}= \begin{cases}1, & |i-j|=1 \\ \frac{\sin \left(\frac{2 \pi}{N}\right)}{\sin \left(\frac{\pi}{N}\right)}, & |i-j|=2\end{cases}
$$

which are the distances between the corresponding vertices for a regular $N$-polygon with unit side length.

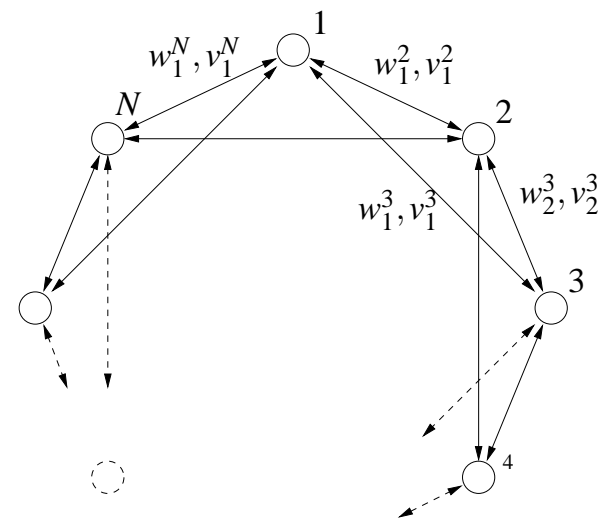

Fig. 3. System topology for Example 1.

Define the inputs to be as in [3] by

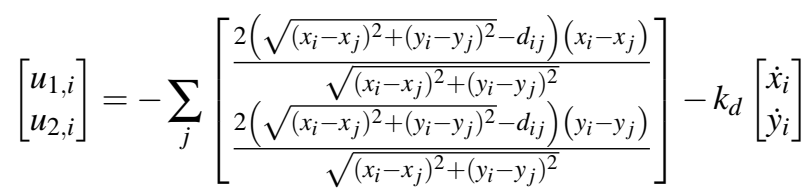

where $k_{d}$ is a positive constant damping gain and $j \in$ $\{i-2, i-1, i+1, i+2\}$.

This is a feedback symmetric system. In detail, by construction the dynamics for each agent are the same, i.e., $f_{i}(x)=f_{j}(x)$ and $g_{j, i}=g_{j, k}$. The index sets are

$$
\mathcal{V}_{i}=\mathcal{W}_{i}=\{i-2, i-1, i+1, i+2\} .
$$

Each agent outputs its position, so $w_{i}^{l}(x)=\hat{f}_{i}^{l}(x)=\left[\begin{array}{ll}x_{i} & y_{i}\end{array}\right]^{T}$. Finally, since the inputs are a function of the state of component $i$ and the components to which it is connected

$$
\left[\begin{array}{l}
u_{1, i} \\
u_{2, i}
\end{array}\right]=\left[\begin{array}{l}
u_{1, i}\left(x_{i}, v_{i}^{i-2}, v_{i}^{i-1}, v_{i}^{i+1}, v_{i}^{i+2}\right) \\
u_{2, i}\left(x_{i}, v_{i}^{i-2}, v_{i}^{i-1}, v_{i}^{i+1}, v_{i}^{i+2}\right)
\end{array}\right] .
$$

Thus, the system satisfies all the elements of the definition of a symmetric feedback system.

Finally, if we order $\mathcal{W}_{i}=\{i-2, i-1, i+2 i+2\}$ for each $i$, the requirement of Equation 4 is satisfied. Hence, a system of size $N+1$ and a system of size $N$ are equivalent.

\section{B. Stability of Symmetric Systems}

Based upon the definition of equivalent symmetric systems, it is possible to show that the stability of a larger symmetric system follows from the stability of a smaller one if the associated Lyapunov function is of a particular form. In this paper we always refer to stability as stability in the sense of Lyapunov.

Proposition 1 Given a feedback symmetric system in one spatial dimension of size $N$, assume that it is stable (resp. asymptotically stable) in the sense of Lyapunov and furthermore that the Lyapunov function is of the form

$$
\begin{aligned}
& V(x)=\sum_{i=1}^{N} V_{i}\left(x_{i}, w_{i-1}^{+}\left(x_{i-1}\right), w_{i-1}^{-}\left(x_{i+1}\right)\right), \\
& \dot{V}(x)=\nabla V \cdot(f(x)+g(x) u(x)) \leq 0,
\end{aligned}
$$


and the $V_{i}$ are symmetric in the sense that

$$
\begin{aligned}
& V_{i}\left(x_{i}, w_{i-1}^{+}\left(x_{i-1}\right), w_{i+1}^{-}\left(x_{i+1}\right)\right)= \\
& \quad V_{j}\left(x_{j}, w_{j-1}^{+}\left(x_{j-1}\right), w_{j+1}^{-}\left(x_{j+1}\right)\right) \quad \forall i, j=1, \ldots, N .
\end{aligned}
$$

Then an equivalent feedback system of size $N+1$ is also stable (resp. asymptotically stable) in the sense of Lyapunov. $\square$

For a proof, see [9]. The idea is that due to the structure of the Lyapunov function given in Equation 5, as long as the larger system is equivalent, then each of the terms in the Lyapunov function corresponding to each agent must be of a similar form. The logic of the proof is analogous to the proofs appearing subsequently in this paper, therefore, the details are omitted.

Remark 1 For the rest of this paper, we will specifically focus on the problem of formation control for a system of multiple robotic agents. Specifically, only the relative position of each agent with respect to the others is controlled and hence any configuration wherein the relative positions are the desired ones will satisfy the formation objective, in other words, the formation is only defined up to an arbitrary rotation and translation. We will assume that there is a function, $V(x) \geq 0$ that is equal to zero only when the robots are in the desired formation. In general, there are an infinite number of configurations that satisfy the formation since the formation can be translated and rotated; hence, LaSalle's invariance principle is the basis for the stability proof. See [3] for a complete exposition.

Example 2 Continuing Example 1, for a fleet of 5 agents, define a Lyapunov function as

$V=\frac{1}{2} \sum_{i=1}^{5}\left[\left(\dot{x}_{i}^{2}+\dot{y}_{i}^{2}\right)+\sum_{j}\left(\sqrt{\left(x_{i}-x_{j}\right)^{2}+\left(y_{i}-y_{j}\right)^{2}}-d_{i j}\right)^{2}\right]$,

where $j \in\{i-2, i-i, i+1, i+2\}$ and $d_{i j}$ is the desired distance between robots defined previously. By construction, this Lyapunov function satisfies the hypothesis of Proposition 1. Computing $\dot{V}$ gives

$$
\begin{aligned}
& \dot{V}=\nabla V \cdot(f+g u) \\
& =\sum_{i=1}^{5}\left[\begin{array}{c}
\sum_{j} 2\left(\frac{\sqrt{\left(x_{i}-x_{j}\right)^{2}+\left(y_{i}-y_{j}\right)^{2}}-d_{i j}}{\sqrt{\left(x_{i}-x_{j}\right)^{2}+\left(y_{i}-y_{j}\right)^{2}}}\left(x_{i}-x_{j}\right)\right) \\
\dot{x}_{i} \\
\sum_{j} 2\left(\frac{\sqrt{\left(x_{i}-x_{j}\right)^{2}+\left(y_{i}-y_{j}\right)^{2}}-d_{i j}}{\sqrt{\left(x_{i}-x_{j}\right)^{2}+\left(y_{i}-y_{j}\right)^{2}}}\left(y_{i}-y_{j}\right)\right) \\
\dot{y}_{i}
\end{array}\right] \\
& \cdot\left[\begin{array}{c}
\dot{x}_{i} \\
-\sum_{j} \frac{2\left(\sqrt{\left(x_{i}-x_{j}\right)^{2}+\left(y_{i}-y_{j}\right)^{2}}-d_{i j}\right)\left(x_{i}-x_{j}\right)}{\sqrt{\left(x_{i}-x_{j}\right)^{2}+\left(y_{i}-y_{j}\right)^{2}}}-k_{d} \dot{x}_{i} \\
-\sum_{j} \frac{2\left(\sqrt{\left(x_{i}-x_{j}\right)^{2}+\left(y_{i}-y_{j}\right)^{2}}-d_{i j}\right)\left(y_{i}-y_{j}\right)}{\sqrt{\left(x_{i}-x_{j}\right)^{2}+\left(y_{i}-y_{j}\right)^{2}}}-k_{d} \dot{y}_{i}
\end{array}\right] \\
& =\sum_{i=1}^{5}-k_{d}\left(\dot{x}_{i}^{2}+\dot{y}_{i}^{2}\right) \text {. }
\end{aligned}
$$

By Proposition 1, this will also hold for any $N$. LaSalle's invariance principle guarantees that the system converges asymptotically to the desired formation. Simulation results for this system are included in [9], and simulations illustrating the extension for robustness will appear subsequently in this paper.

Two issues that are encompassed by the framework developed here but not addressed in detail are collision avoidance and uniqueness of the formation. Both are addressed in [3] and are equivalently handled by the approach in this paper.

\section{Formation Robustness Under AgEnt FAilures}

This section presents the main contributions of this paper, both of which, as will be demonstrated subsequently, follow from logic similar to the proof of Proposition 1. In this paper the result is limited to the formation control problem, but the results are clearly of general applicability. First we define a formation Lyapunov function.

Definition 2 Let $x \in \mathbb{R}^{m \times n}$ denote the configuration of a set of $m$ agents. Let $V: \mathbb{R}^{m \times n} \rightarrow \mathbb{R}$ be continuously differentiable with $V(x)=0$ when the agents are in the desired relative formation. If the largest invariant set of points in $\mathbb{R}^{m \times n}$ is equal to the the set of points where $V(x)=0$, then $V(x)$ is a formation Lyapunov function.

The following proposition contains two parts. First, given a formation Lyapunov function, if one agent fails in a manner such that it stays at rest, then the formation is still asymptotically stable. Second, if more than one agent fails in a manner in which their velocities are zero, the system is stable in the sense of Lyapunov. Stable in the sense of Lyapunov does not imply asymptotic convergence to the formation, but rather loosely speaking, is a guarantee that the formation does not blow up. For a generic system, this latter property is not necessarily apparent a priori.

Proposition 2 Given a feedback symmetric system in one spatial dimension of size $N$, and a formation Lyapunov function of the form

$$
\begin{aligned}
& V(x)=\sum_{i=1}^{N} V_{i}\left(x_{i}, w_{i-1}^{+}\left(x_{i-1}\right), w_{i-1}^{-}\left(x_{i+1}\right)\right), \\
& \dot{V}(x)=\nabla V \cdot(f(x)+g(x) u(x)) \leq 0,
\end{aligned}
$$

if the $V_{i}$ are symmetric in the sense that

$$
\begin{aligned}
& V_{i}\left(x_{i}, w_{i-1}^{+}\left(x_{i-1}\right), w_{i+1}^{-}\left(x_{i+1}\right)\right)= \\
& \quad V_{j}\left(x_{j}, w_{j-1}^{+}\left(x_{j-1}\right), w_{j+1}^{-}\left(x_{j+1}\right)\right) \quad \forall i, j=1, \ldots, N
\end{aligned}
$$

then

1) if the only one agent fails in such a manner that $\dot{x}_{i}(t)=$ 0 , then the formation is still asymptotically stable;

2) if multiple agents fail in such a manner that $\dot{x}_{i}(t)=0$ for $i \in\left\{i_{1}, \ldots, i_{p}\right\}$, then the system is stable in the sense of Lyapunov.

If either case is true for a system with $N$ agents, then it also holds for an equivalent symmetric system with $M>N$ agents. 
Proof: First we consider the nature of a symmetric system and Lyapunov function. Then we will consider the two cases.

By direct computation

$$
\begin{aligned}
\dot{V}= & \sum_{i=1}^{N} \dot{V}_{i} \\
= & \sum_{i=1}^{N} \sum_{j=1}^{N} \sum_{k=1}^{m} \frac{\partial V_{i}}{\partial x_{j}}\left[f_{j}\left(x_{j}\right)\right. \\
& \left.+g_{j, k}\left(x_{j}\right) u_{j, k}\left(x_{j}, w_{j+1}^{-}\left(x_{j+1}\right), w_{j-1}^{+}\left(x_{j-1}\right)\right)\right] \\
= & \sum_{i=1}^{N} \sum_{j=i-1}^{i+1} \sum_{k=1}^{m} \frac{\partial V_{i}}{\partial x_{j}}\left[f_{j}\left(x_{j}\right)\right. \\
& \left.+g_{j, k}\left(x_{j}\right) u_{j, k}\left(x_{j}, w_{j+1}^{-}\left(x_{j+1}\right), w_{j-1}^{+}\left(x_{j-1}\right)\right)\right] .
\end{aligned}
$$

By the assumption of symmetry in $V$ and the fact that the system is symmetric,

$$
\begin{aligned}
\dot{V}= & N \sum_{j=N, 1,2} \sum_{k=1}^{m} \frac{\partial V_{i}}{\partial x_{j}}\left[f_{j}\left(x_{j}\right)\right. \\
& \left.+g_{j, k}\left(x_{j}\right) u_{j, k}\left(x_{j}, w_{j+1}^{-}\left(x_{j+1}\right), w_{j-1}^{+}\left(x_{j-1}\right)\right)\right]
\end{aligned}
$$

for each $i \in\{1, \ldots, N\}$. Since $\dot{V}(t) \leq 0$,

$$
\begin{aligned}
& \sum_{j=N, 1,2} \sum_{k=1}^{m} \frac{\partial V_{i}}{\partial x_{j}}\left[f_{j}\left(x_{j}\right)\right. \\
& \left.\quad+g_{j, k}\left(x_{j}\right) u_{j, k}\left(x_{j}, w_{j+1}^{-}\left(x_{j+1}\right), w_{j-1}^{+}\left(x_{j-1}\right)\right)\right] \leq 0 .
\end{aligned}
$$

By the same construction, for the equivalent system with $M>N$ agents

$$
\begin{aligned}
\dot{V}=M \sum_{j=N, 1,2} & \sum_{k=1}^{m} \frac{\partial V_{i}}{\partial x_{j}}\left[f_{j}\left(x_{j}\right)\right. \\
& \left.+g_{j, k}\left(x_{j}\right) u_{j, k}\left(x_{j}, w_{j+1}^{-}\left(x_{j+1}\right), w_{j-1}^{+}\left(x_{j-1}\right)\right)\right]
\end{aligned}
$$

for each $i \in\{1, \ldots, M\}$, which is less than or equal to zero by Equation 7 .

Now consider the two cases.

1) Since $V(x)$ is a formation Lyapunov function, for a fixed $x_{i}$ there exist formations such that $V(x)=0$. Due to the assumed symmetry of $V$, i.e., $V=\sum_{j} V_{j}$, then $\dot{V}=\sum_{j} \dot{V}_{j}$. With the failure of one agent, if $\dot{x}_{i}=0$, then it follows from the second line of Equation 6 that $\dot{V}_{i}=0$ and hence $\dot{V}=\sum_{j \neq i} \dot{V}_{j}$. However, this is still negative semi-definite due to Equation 5 and by LaSalle's invariance principle, the system converges asymptotically to the desired formation.

2) In this case, assume agents $i_{1}, i_{2}, \ldots, i_{p}$ failed in the indicated manner. Then $\dot{V}=\sum_{j} \dot{V}_{j}=\sum_{j \neq i_{1}, \ldots, i_{p}} V_{i}$. As before, $\dot{V} \leq 0$ which implies stability in the sense of Lyapunov.

Observe that the proof for the first part depends on LaSalle's invariance principle but that the proof for the second does not. This is due to the fact that the first result is for asymptotic stability to the desired formation while the latter is simply stability in the sense of Lyapunov. In the latter

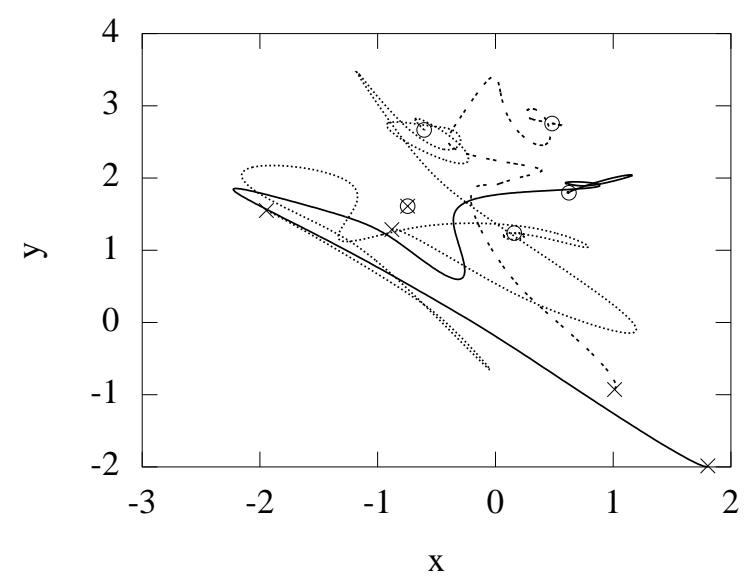

Fig. 4. Simulation results illustrating formation robustness with the failure of one agent.

case, appealing to the invariance principle is not needed. Consequently, the former guarantees asymptotic convergence to the desired formation; whereas, the latter does not. In fact, the latter cannot possibly asymptotically converge to the formation unless the initial conditions for the agents that failed are in the desired formation. The result is still useful, however, because it guarantees that the control law will not cause the formation to blow up when an arbitrary number of agents fails.

The following example demonstrates the result.

Example 3 Continuing from Example 2, assume that the first agent fails in a manner such that $\dot{x}_{1}=0$. This could happen, for example, if all the inputs fail and the agent starts from rest, or, more realistically, a fault-detection algorithm in the agent commands it to stop upon the event of some failure. All the requirements of Proposition 2 are met, and thus the formation must still be asymptotically stable. Figure 4 illustrates the trajectories for simulation results in this case where the initial condition for each agent is indicated with an $x$ and the final configuration for each agent is marked with a $\circ$, illustrating the fact that the formation objective is, in fact, met even though one agent remains stationary. Furthermore, the formation will be stable for any $N>5$.

The following example demonstrates the second result.

Example 4 Continuing from Example 3, assume that the first and second agents fail in a manner such that they remain stationary. Proposition 2 are met, and thus the formation must still be stable. Figure 5 illustrates the trajectories in this case with the initial conditions marked with an $\times$ and the final configurations marked with an $\circ$. The formation does not asymptotically converge to the desired formation; however, it is still stable in the sense of Lyapunov. The robots that failed are located approximately at the top center and right center of the figure. 


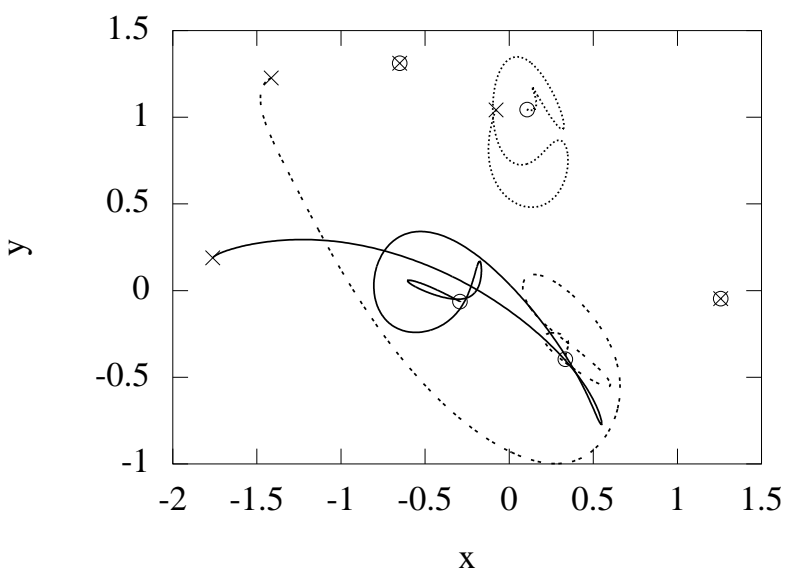

Fig. 5. Simulation results illustrating formation robustness with the failure of two agents.

\section{CONCLUSIONS}

This paper develops generally-applicable conditions for stability of formations of multiple robotic vehicles when robots fail. Under the specified conditions, when one agent fails, the formation is still asymptotically stable. Furthermore, when more than one agent fails, the system is still stable in the sense of Lyapunov. Also, at the cost of symmetry and the ability to decompose $V$ given in Equation 5, the results apply to any system with an arbitray number of agents greater than $N$. It is based upon our prior work in [9] which was directed toward spatially periodic systems "built-up" from periodically interconnected components. Observing that many of the formation control algorithms in the literature are not limited by the number of components, but often are limited by assuming specific dynamics, the main contribution of this paper is to formulate a theoretical framework in which stability of many distributed systems can be considered. The result was demonstrated using two different failure scenarios. Generalizing the results to allow for only a limited number of the components to be symmetric resulting in, for example, line formations, is the subject of current research efforts.

Current efforts are also directed toward the problem of boundedness. This arises in the context of formation control when, for example, in surveillance problems where the region corresponding to each agent defines a formation, but each agent is needed to explore that region. In such a case, time varying inputs may be added to the formation control system. Simulations for the same example considered in this paper indicate that such systems have bounded solutions, as is illustrated in Figure 6, where the ellipses are the steady state responses due to additional forces on each robot given by $f_{x}(t)=0.25 \sin t$ and $f_{y}=0.5 \cos t$. A system of this type would be expected to have a response that has bounded deviations from the desired formation. However, proving the result is difficult because asymptotic convergence is to a set rather than the origin. Hence, proving boundedness will require considering the dynamics on a quotient space and will be the focus of a future publication.

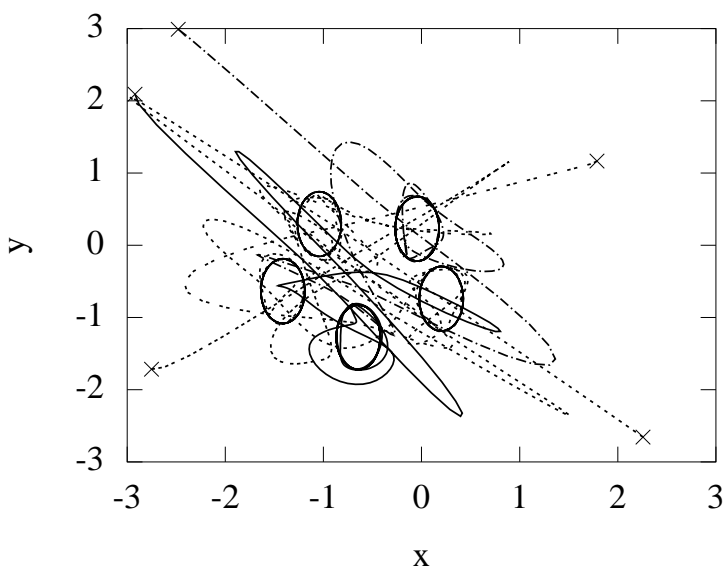

Fig. 6. Simulation results illustrating bounded formation solutions.

\section{REFERENCES}

[1] E. Rimon and D. E. Koditschek, "Exact robot navigation using artificial potential functions," IEEE Transactions on Robotics and Automation, vol. 8, no. 5, pp. 501-518, 1992.

[2] N. Leonard and E. Fiorelli, "Virtual leaders, artificial potentials, and coordinated cont," 2001, pp. 2968-2973.

[3] R. Olfati-Saber and R. M. Murray, "Distributed cooperative control of multiple vehicle formations using structural potential functions," in Proceedings of the 2002 IFAC World Congress.

[4] R. M. Murray, "Recent research in cooperative control of multivehicle systems," Journal of Dynamic Systems, Measurement, and Control, vol. 129, no. 5, pp. 571-583, 2007.

[5] D. Swaroop and J. K. Hedrick, "String stability of interconnected systems," IEEE Transactions on Automatic Control, vol. 41, no. 3, pp. 349-357, 1996.

[6] J. Ousingsawat and M. E. Campbell, "Establishing trajectories for multi-vehicle reconnaissance," in AIAA Conference on Guidance, Navagation and Control, 2004

[7] T. Shima, S. Rasmussen, A. Sparks, and K. Passino, "Multiple task assignments for cooperating uninhabited aerial vehicles using genetic algorithms," Computers and Operational Research, vol. 33, no. 11, pp. 3252-3269.

[8] P. Ögren, M. Egerstedt, and X. Hu, "A control lyapunov function approach to multiagent coordination," IEEE Transactions on Robotics and Automation, vol. 18, no. 5, pp. 847-851, October 2002.

[9] B. Goodwine and P. Antsaklis, "Multiagent coordination exploiting system symmetries," in Proceedings of the 2010 American Controls Conference, Baltimore, MD, 2010.

[10] R. DÁndrea and G. E. Dullerud, "Distributed control design for spatially interconnected systems," IEEE Transactions on Automatic Control, vol. 48, no. 9, pp. 1478-1495, September 2003.

[11] B. Recht and R. DÁndrea, "Distributed control of systems over discrete groups," IEEE Transactions on Automatic Control, vol. 49, no. 9, pp. 1446-1452, September 2004.

[12] M. B. McMickell and B. Goodwine, "Reduction and nonlinear controllability of symmetric distributed systems," International Journal of Control, vol. 76, no. 18, pp. 1809-1822, 2003.

[13] — , "Reduction and controllability of robotic systems with drift," in Proceedings of the 2002 IEEE International Conference on Robotics and Automation, 2002.

[14] _ , "Motion planning for symmetric distributed robotic systems," in 2003 IEEE International Conference on Robotics and Automation, 2003.

[15] _ _ "Motion planning for nonlinear symmetric distributeed robotic systems," International Journal of Robotics Research, vol. 26, no. 10, pp. 1025-1041, October 2007.

[16] M. B. McMickell, "Reduction and control of nonlinear symmetric distributed robotic systems," Ph.D. dissertation, University of Notre Dame, 2003. 